\title{
Novel Automatic Detection of Pleura and B-lines (Comet-Tail Artifacts) on In-Vivo Lung Ultrasound Scans.
}

Moshavegh, Ramin; Hansen, Kristoffer Lindskov; Møller-Sørensen, Hasse; Hemmsen, Martin Christian; Ewertsen, Caroline; Nielsen, Michael Bachmann; Jensen, Jørgen Arendt

\section{Published in:}

Proceedings of SPIE

Link to article, DOI:

$10.1117 / 12.2216499$

Publication date:

2016

Document Version

Peer reviewed version

Link back to DTU Orbit

Citation (APA):

Moshavegh, R., Hansen, K. L., Møller-Sørensen, H., Hemmsen, M. C., Ewertsen, C., Nielsen, M. B., \& Jensen, J. A. (2016). Novel Automatic Detection of Pleura and B-lines (Comet-Tail Artifacts) on In-Vivo Lung Ultrasound Scans. In N. Duric, \& B. Heyde (Eds.), Proceedings of SPIE (Vol. 9790). [97900K] SPIE - International Society for Optical Engineering. https://doi.org/10.1117/12.2216499

\section{General rights}

Copyright and moral rights for the publications made accessible in the public portal are retained by the authors and/or other copyright owners and it is a condition of accessing publications that users recognise and abide by the legal requirements associated with these rights.

- Users may download and print one copy of any publication from the public portal for the purpose of private study or research.

- You may not further distribute the material or use it for any profit-making activity or commercial gain

- You may freely distribute the URL identifying the publication in the public portal 


\title{
Novel Automatic Detection of Pleura and B-lines (Comet-Tail Artifacts) on In-Vivo Lung Ultrasound Scans
}

\author{
Ramin Moshavegh ${ }^{a}$, Kristoffer Lindskov Hansen $^{b}$, Hasse Møller Sørensen ${ }^{c}$ \\ Martin Christian Hemmsen ${ }^{a}$, Caroline Ewertsen ${ }^{b}$, Michael Bachmann Nielsen ${ }^{b}$, and \\ Jørgen Arendt Jensen ${ }^{a}$ \\ ${ }^{a}$ Center for Fast Ultrasound Imaging, Dept. of Elec. Eng., Technical University of Denmark, \\ DK-2800 Lyngby, Denmark \\ ${ }^{b}$ Dept. of Radiology, ${ }^{c}$ Dept. of Thoracic anestesiologic, Copenhagen University Hospital, \\ DK-2100 Copenhagen, Denmark
}

\begin{abstract}
This paper presents a novel automatic method for detection of B-lines (comet-tail artifacts) in lung ultrasound scans. B-lines are the most commonly used artifacts for analyzing the pulmonary edema. They appear as laser-like vertical beams, which arise from the pleural line and spread down without fading to the edge of the screen. An increase in their number is associated with presence of edema. All the scans used in this study were acquired using a BK3000 ultrasound scanner (BK Ultrasound, Denmark) driving a 192-element 5.5 MHz wide linear transducer (10L2W, BK Ultrasound). The dynamic received focus technique was employed to generate the sequences. Six subjects, among those three patients after major surgery and three normal subjects, were scanned once and Six ultrasound sequences each containing 50 frames were acquired. The proposed algorithm was applied to all 300 in-vivo lung ultrasound images. The pleural line is first segmented on each image and then the B-line artifacts spreading down from the pleural line are detected and overlayed on the image. The resulting 300 images showed that the mean lateral distance between B-lines detected on images acquired from patients decreased by $20 \%$ in compare with that of normal subjects. Therefore, the method can be used as the basis of a method of automatically and qualitatively characterizing the distribution of B-lines.
\end{abstract}

Keywords: B-lines, comet-tail artifacts, segmentation, lung disease, pulmonary edema, ultrasound imaging

\section{INTRODUCTION}

In ultrasound an intimate mixture of air and water can characterize the lung. The change in their balance can be the sign of the pulmonary diseases. Interactions of water and air in lung ultrasound scans generate a variety of artifacts, and therefore, the lung ultrasound of pulmonary disease is rather based on analysing these artifacts than pure visualization of the structures. B-lines are laser-like vertical artifacts, which start from the pleura and spread down to the edge of the screen. B-line detection is an essential parameter in the assessment of lung-edema in lung ultrasound imaging, which is often present in patients with heart and lung problems as well as patients after major surgery. ${ }^{1-3}$ B-line detection is also used in detection of pneumothorax as B-lines disappear, when a pneumothorax is present. The condition where air is found within the pleural cavity can result in collapsed lung, and if untreated be fatal. In daily clinical practice, patients suspected for lung edema or pneumothorax are imaged with X-ray, and often repeatedly imaged with short intervals to monitor the effect of the applied treatment. Lung ultrasound is an emerging ultrasound modality, but is often bypassed, as the medical staff handling these patients are not familiar with ultrasound. If ultrasound is used for evaluation of the lungs, the common practice for diagnosing pulmonary edema is based on visual analysis and interpretation of B-lines on still lung ultrasound images. For the detection of pneumothorax, the detection of a single B-line excludes pneumothorax in the imaged lung segment. Fig. 1 illustrates how B-lines are generated. They are reverberation artifacts that originate from water-thickened pulmonary interlobular septa. ${ }^{1}$

Further author information: Ramin Moshavegh, E-mail: ramosh@elektro.dtu.dk 

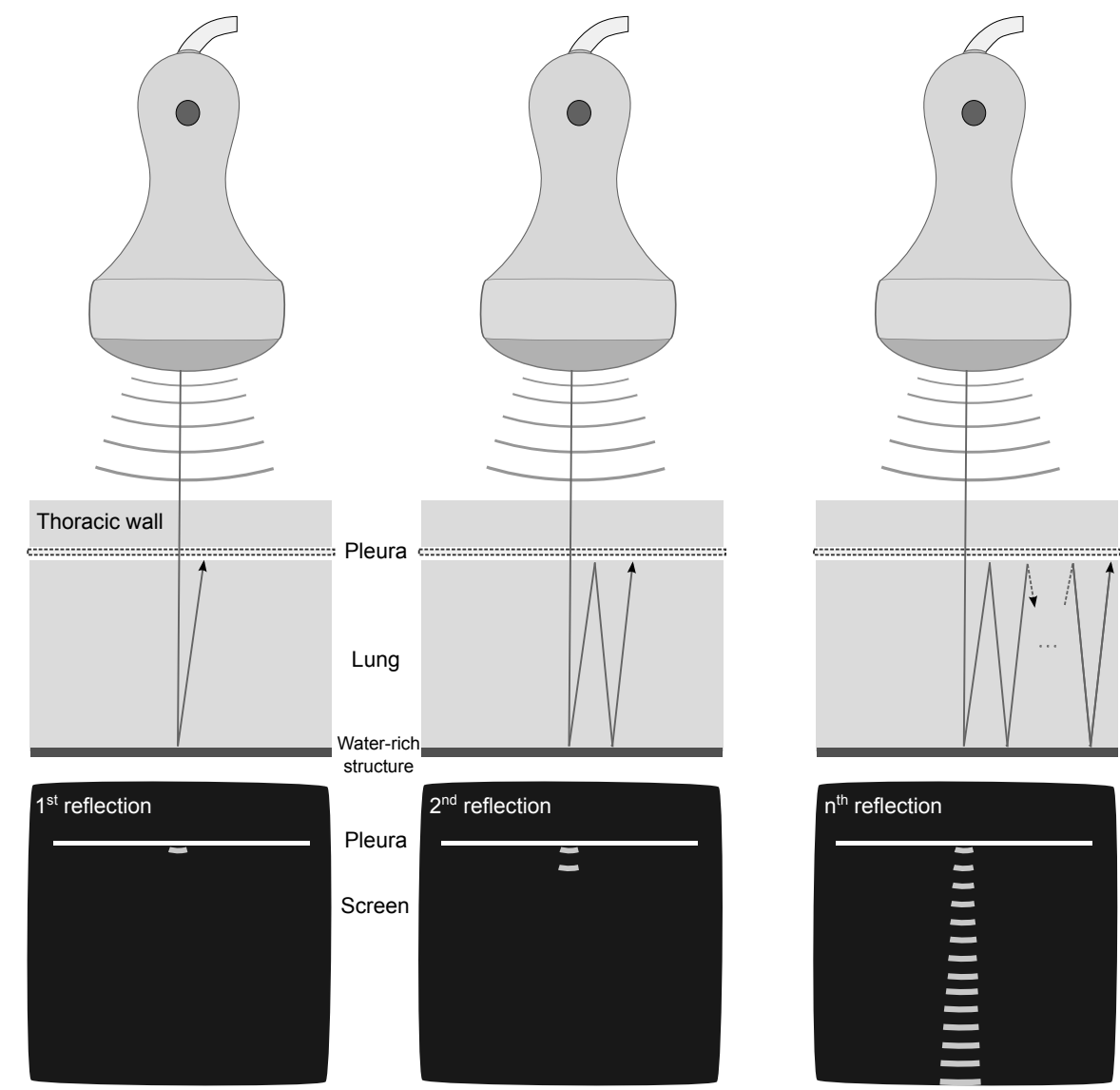

Figure 1: Illustration of how the comet-tail artifacts are generated. They are hyper-echoic reverberation artifacts arising from the pleural line and spreading down towards the lower edge of the screen.

Based on the authors' knowledge, there are no computerized and automated model for characterizing the B-line artifacts in the literature. This paper proposes a novel algorithm as the first possible solution for monitoring of lung edema and detection of pneumothorax. The remainder of this paper is organized as follows. Section 2 introduces the proposed algorithm. Section 3 presents the in-vivo results and discusses the findings. Finally section 4 is the conclusion and the perspectives.

\section{MATERIALS AND METHODS}

This paper introduces an automatic real-time method for detection of pleura and the B-lines in lung ultrasound scans. The method first delineates the pleural line on the image and then detects the B-lines, as they originate from the pleural line and extend to the edge of the display. The proposed algorithm contains four distinct steps. First, the pleural line is delineated using a random walks method. ${ }^{4}$ Second, the upper-pleural region is excluded from the scan, the Hilbert transform of the remaining region is computed, a cumulative frequency histogram is generated, a zone of influence of strong signals in the image is calculated, and the B-line artifacts are identified on the scan plane. Third, an alternate sequential filtering is applied to the results of step 2. Finally, the result of step 3 is top-hat filtered and the B-lines are extracted and overlaid on the B-mode scan. Four main steps of the algorithm are discussed in detail in this section.

\subsection{Step I. Confidence map estimation and delineation of the pleural line}

The first step in detection of the B-lines is to delineate the pleural line on the lung scans. For this purpose a graph based approach that computes a per-pixel uncertainty map based on the information depicted by ultrasound 

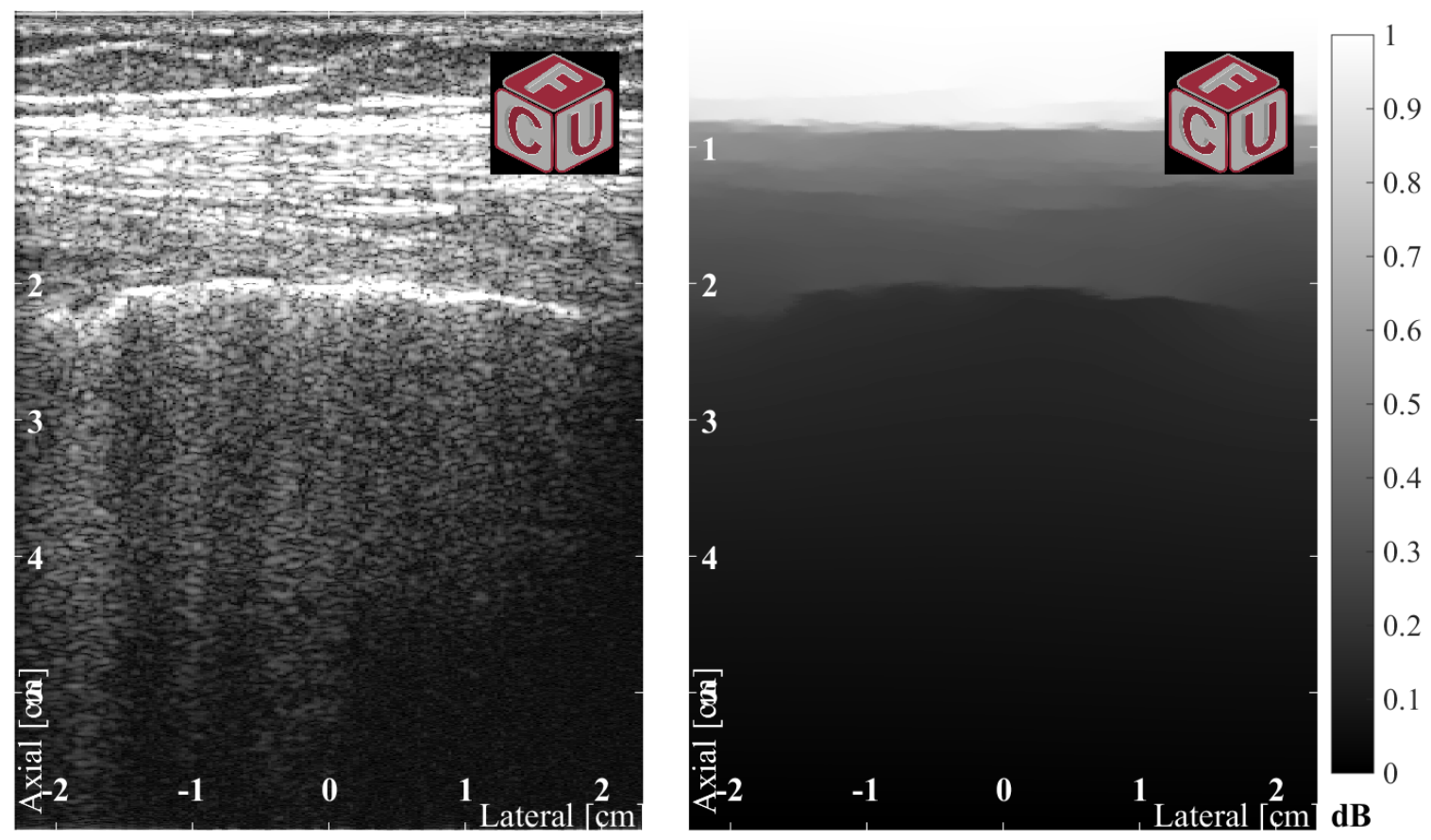

Figure 2: Example of a confidence map computed for a human lung scan. This map is then thresholded to delineate the pleural line.

image is used. ${ }^{5}$ This rather specifically measures the uncertainty in attenuated and/or shadow regions. To identify the map for each ultrasound frame, a random walks framework by taking into account ultrasound specific constraints is used. ${ }^{5}$ The solution to the random walks equilibrium problem is global and takes the entire content of the RF data into account. The required starting points are automatically placed at the beginning of each scan-line to represent the transducer elements. Thus, a problem is formulated by computing the probability of a random walk starting from a pixel to reach the transducer elements. The domain specific knowledge for ultrasound is integrated with a simple modelling of the ultrasound image formation process. The ultrasound specific constraints obtained from physics of acquired RF data by the transducer must be integrated to ensure the accuracy of the confidence map. One of the fundamental properties of ultrasound is the attenuation of the signal by increasing depth. The Beer-Lambert Law is used to express the depth dependent attenuation, and the attenuated signal $I$ could be given by $I=I_{0} \exp (-\alpha d)$, where $I_{0}$ is the initial intensity, $\alpha$ the attenuation coefficient, and $d$ the distance from the source. This property is included into the confidence map in a way that the further away a random walk starts from the transducer is, the more unlikely it will be able to reach one of the transducer elements. The computed confidence map is used to determine the upper-pleural region and exclude that from the Rf-data prior to detecting the B-lines (see Fig. 2). The pleural line is delineated on the confidence map by thresholding the confidence map to be higher than the global threshold of the entire map. The region with values higher than the threshold is considered the upper pleura, and the rest is lower pleural region. In the next step the B-lines starting from the pleura and appearing inside the lower pleural region are detected.

In the next step of the algorithm, after delineating the pleural line in each scan, the B-lines are characterized from the Hilbert transform of the gradient of the RF-data.

\subsection{Step II. B-line detection}

The RF-data corresponding to the region above the pleural line are excluded, and only the RF-data belonging to the lower pleural region is used for B-line detection. This is performed knowing that the artifacts arise from the pleural line and spreads down in the scan. The axial gradient of RF-data is then computed. The Hilbert transform of the axial gradient components is calculated, the absolute value of the complex data is computed and data is compressed by taking the logarithm transform. Finally, a binary mask including the strong specular 
regions inside the compressed envelope data is computed. This binary mask also outlines the most prominent B-lines in the envelope data (see Fig. 3a). The mask is later manipulated in the sections 2.3 and 2.4 to extract the position of the B-lines.

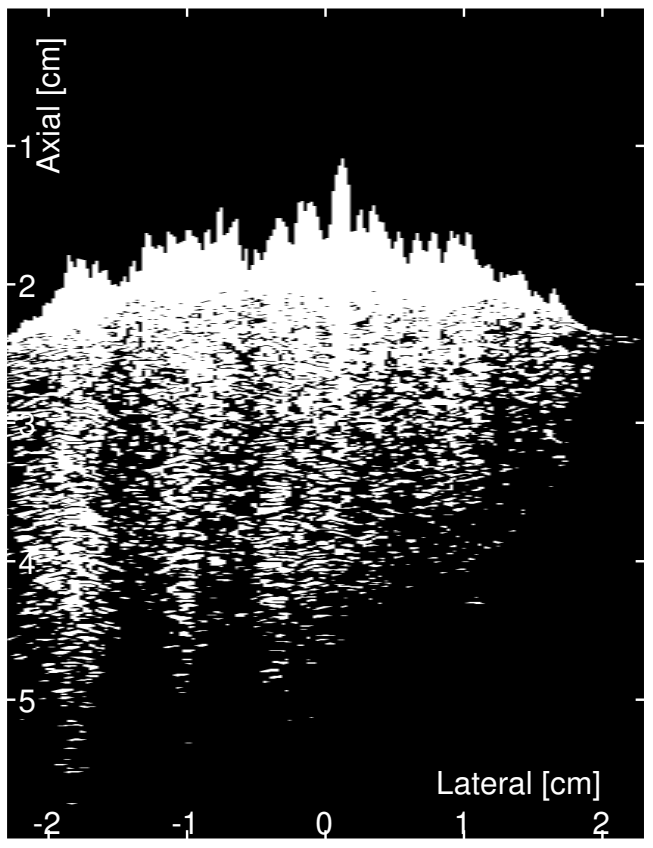

(a) Mask outlining the most prominent B-lines in the compressed data is then computed from the RF-data.

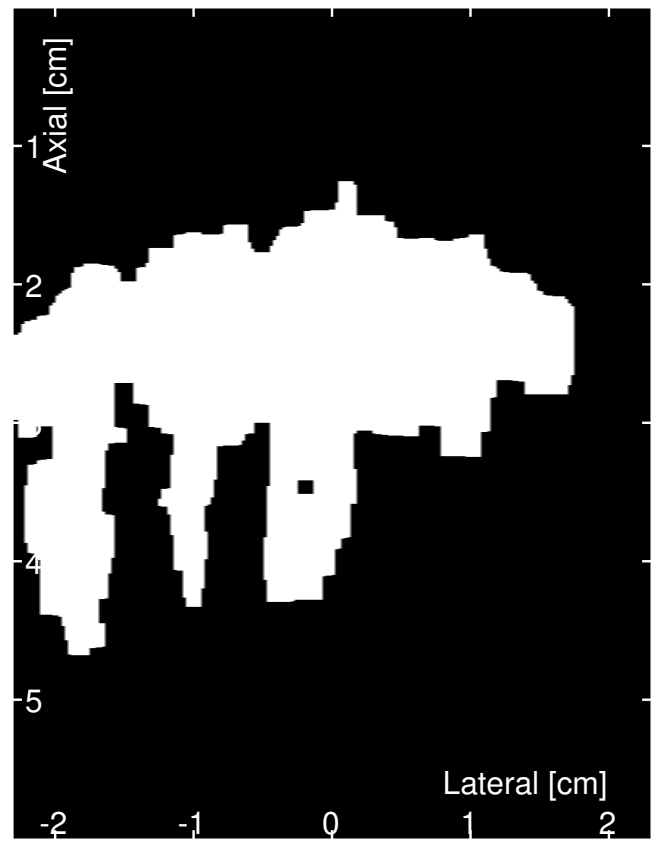

(b) Alternate sequential filtering applied to the mask (a). B-lines are better identified.

Figure 3: Illustration of how B-lines are detected from the RF-data.

\subsection{Step III. Alternate sequential filtering}

An alternate sequential filtering (ASF) procedure using a repeated sequential morphological opening and closing is applied to the mask and the ASF mask is generated (see Fig. 3b). The procedure is depicted in the Alg. 1. The structuring element used in this process is considered to be an axial line-structuring element. The line structuring element along the ultrasound beem is used to ensure that only elongated and axial information (B-line artifacts) in the compressed data is preserved and highlighted.

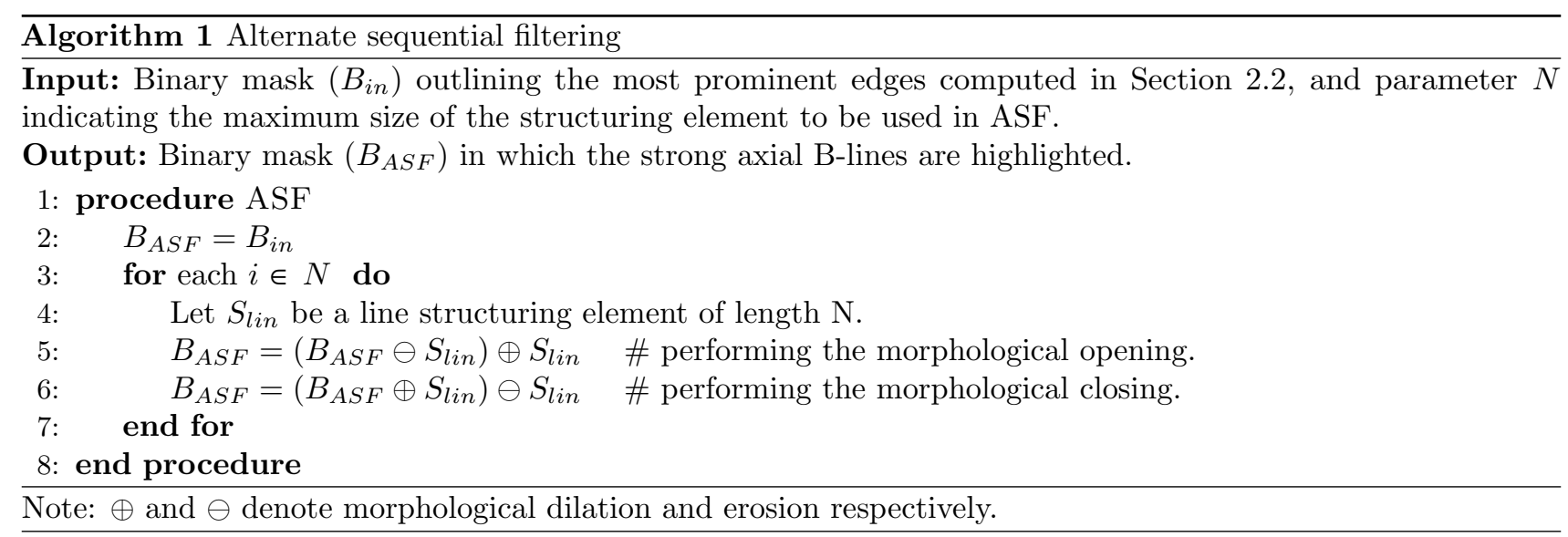




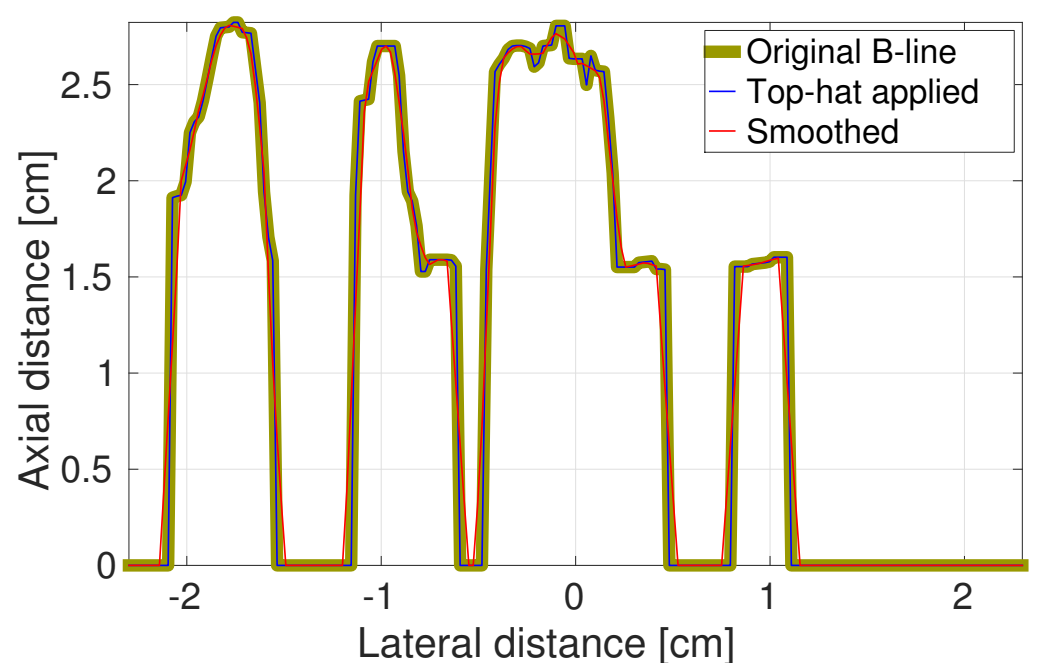

Figure 4: Alternate sequential filtering and the result of the top-hat filtering.

\subsection{Step IV. Top-hat filtering}

The generated mask in the previous step contains prominent axially elongated and adjacent tails locating the B-lines. However, the tails can be laterally connected and that makes the clean separation of them (B-lines) quite difficult. To separate and extract the B-lines automatically, a top-hat filtering procedure is used. The top-hat transform originally proposed by Mayer ${ }^{6}$ is a mathematical morphology operator that uses morphological opening or closing for extracting bright (respectively dark) objects from an uneven background in a 2D grey-scale image. Top-hat transformation can be formulated in two ways: White Top-hat $(W T H)$ and Black Top-hat $(B T H)$. White Top-hat can also be used to identify prominent peaks in a $1 \mathrm{D}$ signal, and the $B T H$ as the dual of the $W T H$ can be used to identify the prominent minima in a 1D signal. In this paper a White Top-hat $(W T H)$ with a flat disk structuring element is used to extract the very the B-lines. The $W T H$ transformation of a $1 \mathrm{D}$ signal $f$ with a flat structuring element $B$ is defined as:

$$
T_{\text {white }}(f)=f-(f \circ B)
$$

First, A curve is generated by computing the axial summation of all pixel values inside mask from Section 2.2. The peaks of the resulting curve are corresponding to the tails locating the B-lines in the mask (see Fig. 4. To identify the peaks of the curve, the Top-hat transform is applied using a flat structuring element $(B)$ that is somewhat larger than the size of the connected regions. The $\circ$ is the opening operator and is basically a min operation that removes the regions smaller than the size of structuring element. Subtracting this signal from the original signal produces a signal that only contains the desired strong peaks. Fig. 4 shows the B-lines detected after alternate sequential filtering and top-hat filtering for a lung scan of a patient with lung edema. The B-lines are overlaid on the corresponding B-mode image in Fig. 5a.

\section{RESULTS AND DISCUSSION}

The proposed algorithm was applied to in-vivo lung scans. Three healthy subjects and three patients with different levels of lung edema were scanned after having major open-chest surgery. A total of 6 lung sequences each containing 50 frames were acquired. The study was approved by the Danish National Committee on Biomedical Research Ethics and the local Ethics Committee, and the volunteers were included into the study after informed consent. Anatomical locations included in the dataset have been analysed first to make sure B-lines exist. The beamformed RF data were acquired using a BK3000 ultrasound scanner (BK Medical, Denmark). A research interface connected to the scanner simultaneously recorded the real-time beamformed RF data from the scanner. ${ }^{7}$ The proposed B-line detection algorithm was applied to the acquired image sequences in MATLAB (2015b). Fig. 5 shows two examples of the detected pleura and B-lines on lung scans of a patient and a normal subject, 
respectively. The mean lateral distance between neighbouring B-lines in the 150 images belonging to the patient is $(0.93 \mathrm{~cm})$ which is decreased by $20 \%$ in compared with that of 150 images belonging normal subjects $(1.17 \mathrm{~cm})$.
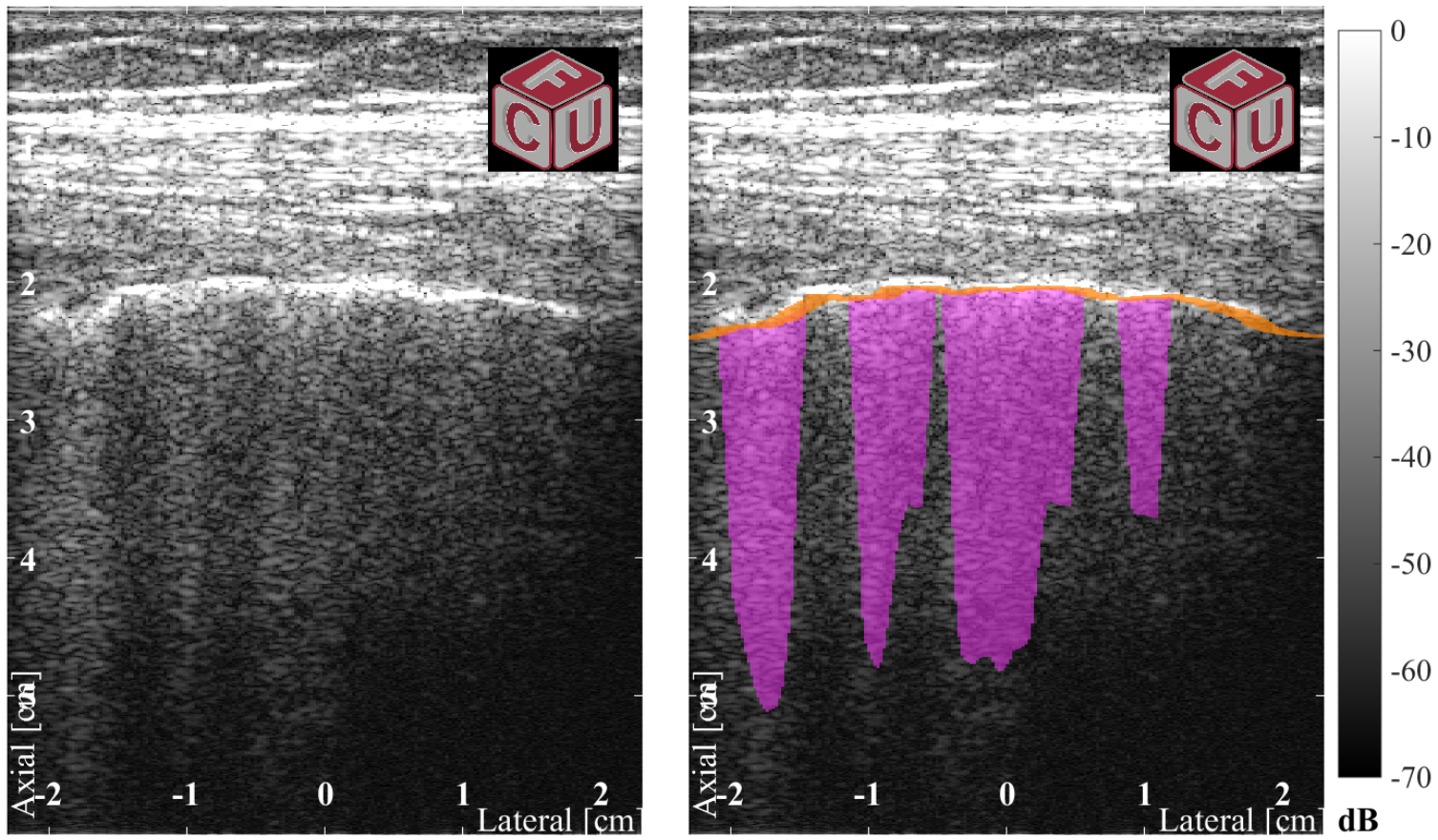

(a) The pleural line outlined and B-lines are overlaid on the lung scan of a patient after surgery.
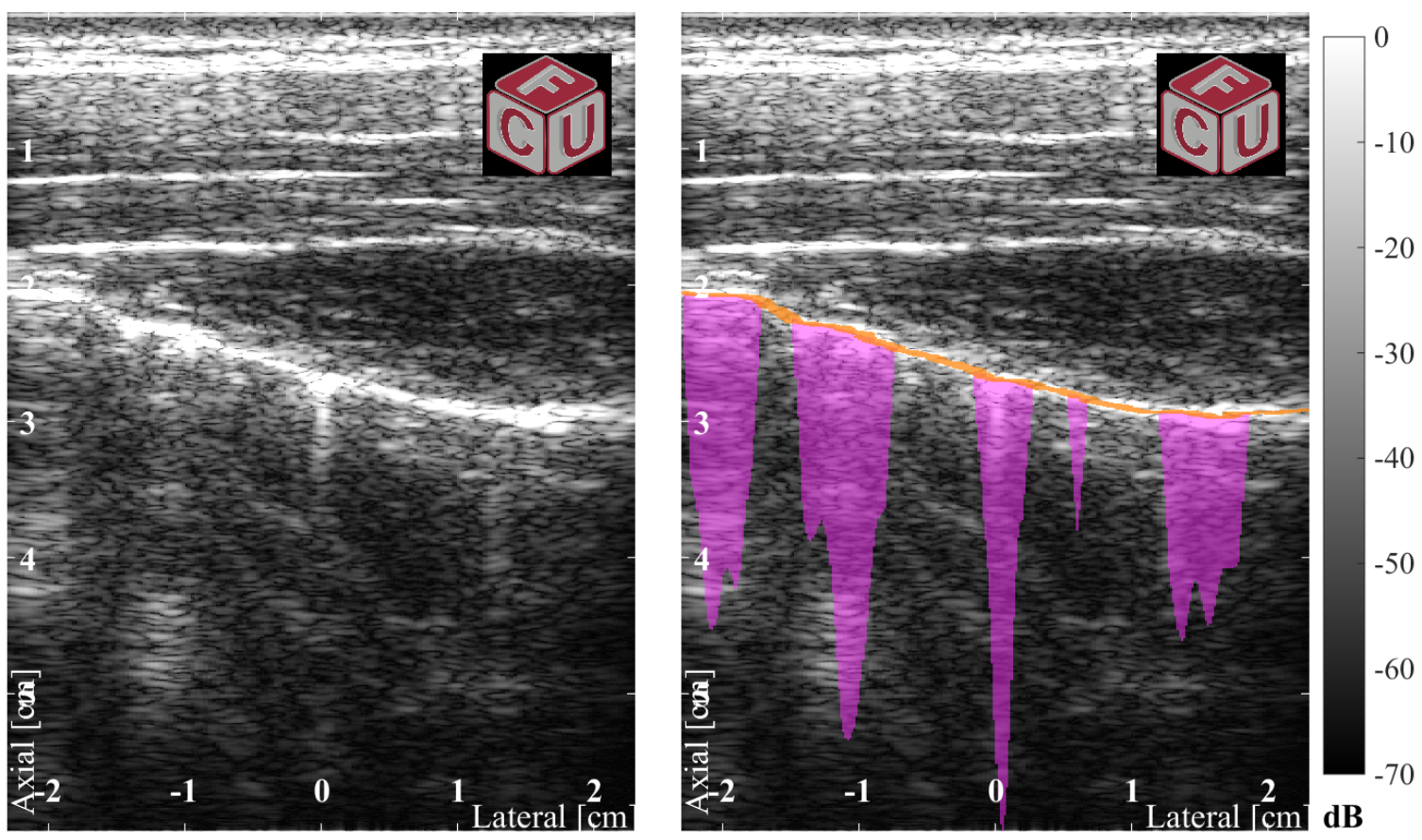

(b) The pleural line outlined and B-lines are overlaid on the lung scan of a normal subject.

Figure 5: Two examples of ultrasound lung scans, on top of which the pleural lines are outlined and B-lines overlaid. 


\section{CONCLUSION}

This paper presented a novel advanced B-line detection algorithm. The novely of the algorithm stems from proposing the first automatic real-time technique for characterization of the B-lines (comet tail artifacts) in lung ultrasound scans as a crucial measure for diagnosing pulmonary edema. The results showed a $20 \%$ decrease in the mean lateral distance between B-lines in patients with lung edema in compare with the normal subjects. Therefore, the proposed method enables the automatic quantitative estimation of edema and have a tremendous clinical impact. The algorithm can also be an aid for the sonographer and a tool for making a quantitative estimation of lung edema and detection of pneumothorax. Furthermore, the algorithm can be an aid for the untrained personnel performing the ultrasound scan, as well as providing a quantitative measure for B-lines presence.

\section{REFERENCES}

[1] Gargani, L., "Lung ultrasound: a new tool for the cardiologist," Cardiovascular ultrasound 9(1), 6 (2011).

[2] Lichtenstein, D. A., "Lung ultrasound in the critically ill," Ann Intensive Care 4(1), 1 (2014).

[3] Volpicelli, G., Mussa, A., Garofalo, G., Cardinale, L., Casoli, G., Perotto, F., Fava, C., and Frascisco, M., "Bedside lung ultrasound in the assessment of alveolar-interstitial syndrome," The American Journal of Emergency Medicine 24(6), 689-696 (2006).

[4] Grady, L., "Random walks for image segmentation," Pattern Analysis and Machine Intelligence, IEEE Transactions on 28, 1768-1783 (Nov 2006).

[5] Karamalis, A., Wein, W., Klein, T., and Navab, N., "Ultrasound confidence maps using random walks," Medical Image Analysis 16(6), 1101-1112 (2012).

[6] Meyer, F., "Contrast features extraction, special issues of practical metallography," in [Quant. Anal. of Microstruct. in Materials Science, Biology and Medicine,], 8 (1977).

[7] Hemmsen, M. C., Nikolov, S. I., Pedersen, M. M., Pihl, M. J., Enevoldsen, M. S., Hansen, J. M., and Jensen, J. A., "Implementation of a versatile research data acquisition system using a commercially available medical ultrasound scanner," IEEE Trans. Ultrason., Ferroelec., Freq. Contr. 59(7), 1487-1499 (2012). 